\title{
Supporting Information for: Accelerating Hydrogen Absorption and Desorption Rates in Palladium Nanocubes with an Ultrathin Surface Modification
}

\author{
Lucy J. T. Metzroth ${ }^{a}$ Elisa M. Miller, ${ }^{a}$ Andrew G. Norman ${ }^{b}$, Sadegh Yazdi,${ }^{c,}{ }^{d}$ and Gerard \\ Michael Carroll ${ }^{a}$ * \\ ${ }^{a}$ Chemistry and Nanoscience Center, National Renewable Energy Laboratory, 15013 Denver \\ West Parkway, Golden, Colorado 80401, United States \\ ${ }^{b}$ Materials Science Center, National Renewable Energy Laboratory, 15013 Denver West \\ Parkway, Golden, Colorado 80401, United States \\ ${ }^{c}$ Renewable \& Sustainable Energy Institute, University of Colorado Boulder, \\ Boulder, Colorado 80309, United States \\ ${ }^{d}$ Materials Science \& Engineering Program, University of Colorado at Boulder, Boulder, \\ Colorado 80309, United States
}

Email: mike.carroll@ nrel.gov

\section{Experimental Section.}

Palladium $\{100\}$ nanocube synthesis. Palladium nanocubes with average dimensions of $17 \mathrm{~nm}$ were synthesized according to previously reported methods. ${ }^{1}$ To an $80{ }^{\circ} \mathrm{C}$ aqueous solution of polyvinylpyrrolidone (PVP), ascorbic acid, and $\mathrm{KBr}$, a solution of $\mathrm{Na}_{2} \mathrm{PdCl}_{4}$ was added to stirred for $3 \mathrm{~h}$. The nanocube product was isolated by centrifugation at 12,000 $\mathrm{g}$ and resuspended in DI water. To remove the terminating PVP surface ligands, we follow previously established procedures $^{2,3}$ where the PVP-capped Pd $\{100\}$ were sonicated in a $1 \mathrm{M} \mathrm{KOH}$ solution for $20 \mathrm{~min}$. Following sonication, the suspension was centrifuged at 12,000 g, the supernatant was decanted, and the pellet and resuspended in $1 \mathrm{M} \mathrm{KOH}$. This process was repeated three to four times, until the remaining Pd $\{100\}$ nanocubes would no longer suspend in DI water.

Electrode Preparation. Unless otherwise stated, the cleaned Pd nanocubes were fabricated into a composite electrode by first preparing a slurry containing the Pd nanocubes, Timcal C65 conductive carbon, and 5\% Nafion 117/ EtOH solution. This slurry was sonicated for $10 \mathrm{~min}$. The slurry was then drop-cast onto the surface of a clean glassy carbon rotating disk electrode and heated in an oven at $150{ }^{\circ} \mathrm{C}$ to remove solvent and dry the composite onto the glassy carbon surface.

Platinum surface functionalization. Platinum was added to the surface of $\operatorname{Pd}\{100\}$ in one of two ways. In the first, a $1 \mu \mathrm{M}$ aqueous solution of $\mathrm{K}_{2} \mathrm{PtCl}_{4}$ was added to a dilute suspension of the Pd $\{100\}$ nanocubes. This mixture was stirred for $24 \mathrm{~h}$, after which, the suspension was centrifuged at 12,000 g, dried, and resuspended in DI water. The centrifugation/resuspension process was repeated three times to remove excess $\mathrm{K}_{2} \mathrm{PtCl}_{4}$. The isolated Pd@Pt $\{100\}$ nanocube product was used for transmission electron microscopy (TEM), scanning transmission electron microscopy (STEM), and STEM energy dispersive x-ray spectroscopy (STEM-EDS) experiments. 
In the second synthetic method, a Pd $\{100\}$ nanocube composite electrode mounted on a rotating disk electrode was dipped into a $1 \mu \mathrm{M}$ solution of $\mathrm{K}_{2} \mathrm{PtCl}_{4}$ while rotating at a rate of $2400 \mathrm{rpm}$ for several minutes. Following this soak, the electrode was rinsed sequentially with $0.1 \mathrm{M} \mathrm{H}_{2} \mathrm{SO}_{4}$, then with DI water. This synthetic method was used to generate samples for electrochemical and XPS experiments. Using suspended Pd NCs and Pd@Pt NCs for TEM and STEM imaging and STEMEDS mapping (as opposed to electrode bound) was performed to ensure that enough space was kept between individual particles which enabled EDS mapping. To ensure that the platinization procedure produces equivalent Pd@Pt surfaces on the electrode and as a suspension, we also performed platinization and TEM measurements on electrode-bound particles. Those images are provided in Figure S5.

Electrochemical Measurements. All electrochemical data were collected in a temperature-controlled electrochemical cell by submerging the cell in an oil bath on top of a heating mantle. The temperature of the oil bath was monitored, and both the oil bath and electrolyte were stirred continuously to ensure a homogeneous thermal profile of the electrolyte. We employ a standard three-electrode configuration consisting of a working, counter, and reference electrode. The working electrode was the Pd nanocube composite mounted on the surface of a glassy carbon rotating disk electrode, the counter electrode was reticulated vitreous carbon separated from the main electrolyte chamber by a porous frit, and the reference was a double junction, saturated $\mathrm{Hg} / \mathrm{HgSO}_{4}$ electrode with a $3 \mathrm{M} \mathrm{K}_{2} \mathrm{SO}_{4}$ solution jacket. The potentials in the main text are reported against the reversible hydrogen electrode which was derived from the Nernst equation. The working electrode was kept at a constant rotation rate of $2400 \mathrm{rpm}$ to eliminate mass transport artifacts of proton delivery from the solution to the electrode surface. The $\mathrm{H}^{+}$limiting current at this concentration and rotation rate was calculated to be $\sim 10 \mathrm{Amps} / \mathrm{cm}^{2}$ (see below). The electrolyte used in these experiments was $0.1 \mathrm{M} \mathrm{H}_{2} \mathrm{SO}_{4}$. Prior to any electrochemical measurement, the solution was purged with $\mathrm{N}_{2}$ for 15 min to remove dissolved oxygen. During measurements, a constant head space of $\mathrm{N}_{2}$ was kept on the electrochemical cell to inhibit $\mathrm{O}_{2}$ solubilization. For the transient measurements in Figure 2c\&d, the current sensitivity channel was kept constant which resulted in a reduced signal-to-noise ratio for the baseline current of Pd@Pt compared to Pd.

During the scan-rate-dependent kinetic measurements, we found that for scan rates faster than $25 \mathrm{mV} / \mathrm{s}$, the $\mathrm{Pd}\{100\}$ nanocubes did not fully hydridate before the reverse scan direction was initiated which resulted in inconsistent measurements. To solve this, during each section of a given $\mathrm{CV}$ scan $\left(\mathrm{H}_{\mathrm{abs}}\right.$ or $\left.\mathrm{H}_{\mathrm{des}}\right)$, we held the electrode potential at the lowest [highest] values for 20 seconds to ensure that before the next sweep was performed, the Pd nanocubes were completely hydridated [dehydridated].

X-ray Photoelectron Spectroscopy. XPS data were obtained on a Physical Electronics 5600 system using Al Ka radiation. The XPS setup was calibrated with Au metal, which was cleaned via Ar-ion sputtering. The raw atomic concentration has a 5\% error due to surface inhomogeneities, surface roughness, literature sensitivity values for peak integration, etc.

Transmission Electron Microscopy/Energy Dispersive X-ray Analysis. TEM samples were prepared by dipping a thin graphene support film TEM grid into a suspension of $\mathrm{Pd}\{100\}$ or Pd@Pt $\{100\}$ nanocubes and drying the sample in air. Data for Figures 1C \& 1D, and Figures S1 $\& S 5$ were obtained on a FEI Tecnai $\mathrm{G}^{2} 30$ SuperTwin TEM operated at $300 \mathrm{kV}$ and a FEI Tecnai F20 UltraTwin field emitting gun (FEG) scanning transmission electron microscope (STEM) 
operated at $200 \mathrm{kV}$. The atomic concentrations listed in Table 1 were measured on a FEI Tecnai F20 UltraTwin STEM and an EDAX Octane T Optima windowless Si drift detector (SDD) EDS system. All STEM images and STEM-EDS maps were obtained on a probe Cs-corrected Titan Themis S/TEM equipped with a SuperX detector (4 SDD detectors) operated at $300 \mathrm{kV}$. HAADFSTEM images were collected at the beam convergence angle of $25 \mathrm{mrad}$ and the collection angle range of 52 to $200 \mathrm{mrad}$.

\section{Self-limiting Pt surface deposition reaction.}

Xia et al, have previously shown that in the absence of $\mathrm{Br}^{-}$and/or a reductant, neither $\mathrm{Pt}^{2+}$ nor $\mathrm{Pt}^{4+}$ replace $\mathrm{Pd}$ in $\mathrm{Pd}\{100\}$ nanocubes. ${ }^{4}$ We find that indeed, $\mathrm{Pt}^{2+}$ does not replace $\mathrm{Pd}$ in the bulk of the nanocube, but does bond to the Pd $\{100\}$ surface and is a self-limiting. Evidence that this functionalization is self-limiting to surface Pd is presented in Figure S1. Here, the CV of the same electrode before, after $5 \mathrm{~min}$, and after $24 \mathrm{~h}$ of soaking in $\mathrm{K}_{2} \mathrm{PtCl}_{4}$ while at a rotation of 2400 rpm shows no change in $\mathrm{H}_{\mathrm{abs}} / \mathrm{des}$ current between $5 \mathrm{~min}$ and $24 \mathrm{~h}$ of soaking. If, for example, $\mathrm{Pt}^{2+}$ performed a continuous galvanic replacement reaction on $\mathrm{Pd}$, the hydrogen storage capacity would diminish to near zero. Instead, what we observe is that the hydrogen storage capacity of the $24 \mathrm{~h}$ soaked electrode is identical to the 5 min-soaked electrode indicating that only a surface modification to the Pd nanocubes occurs.

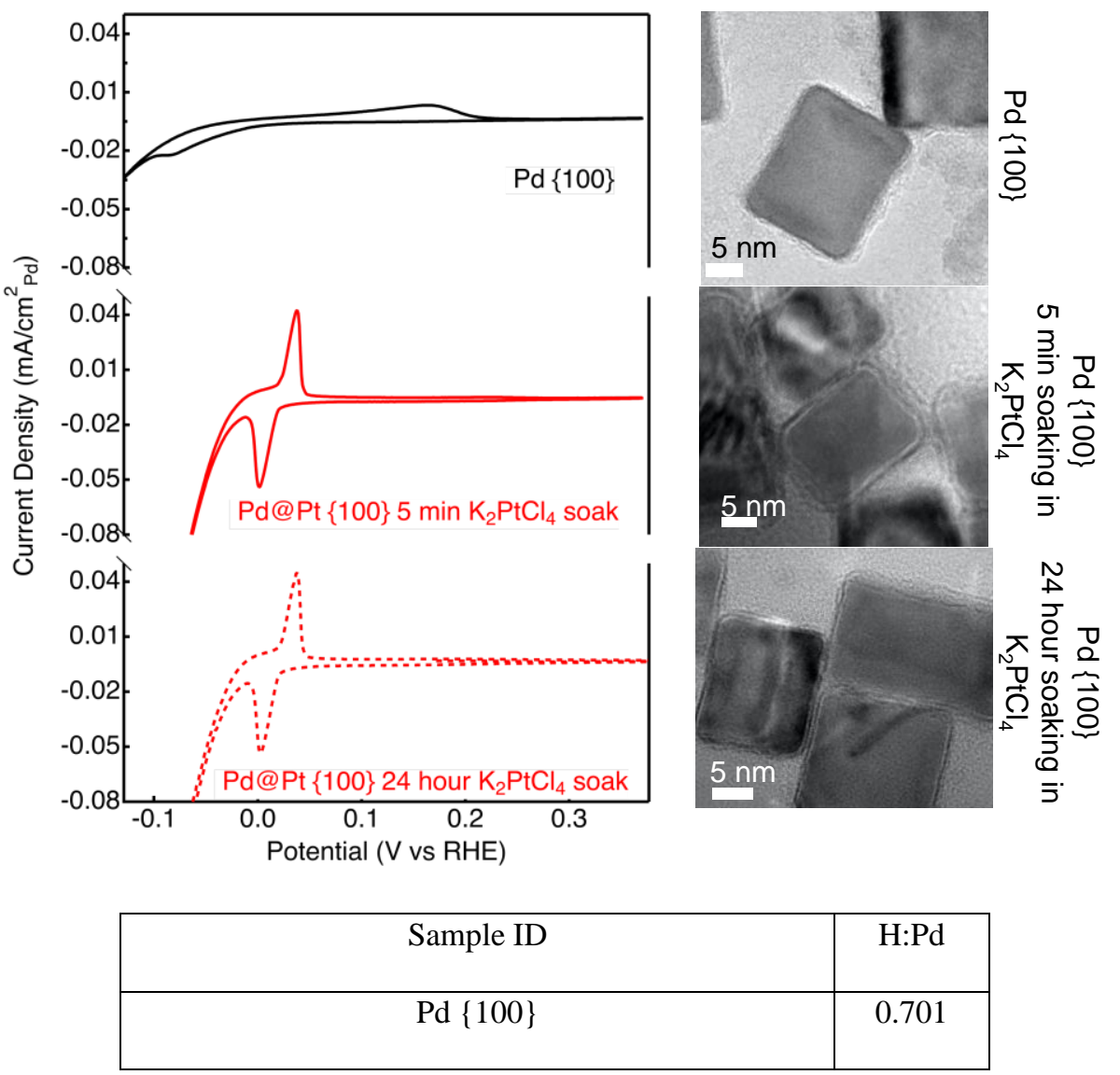




\begin{tabular}{|c|c|}
\hline $\mathrm{Pd} @ \mathrm{Pt}\{100\} 5$ min $\mathrm{K}_{2} \mathrm{PtCl}_{4}$ soak & 0.699 \\
\hline $\mathrm{Pd} @ \mathrm{Pt}\{100\} 24$ hour $\mathrm{K}_{2} \mathrm{PtCl}_{4}$ soak & 0.703 \\
\hline
\end{tabular}

Figure S1. The effect of soaking the $\mathbf{P d}\{\mathbf{1 0 0}\}$ in $\mathbf{K}_{2} \mathbf{P t C l}$. (left) Cyclic voltammograms for a $\mathrm{Pd}\{100\}$ electrode in $0.1 \mathrm{M} \mathrm{H}_{2} \mathrm{SO}_{4}$ (black) before exposure to $\mathrm{K}_{2} \mathrm{PtCl}_{4}$, after $5 \mathrm{~min}$ (red) and $24 \mathrm{~h}$ (red dashed) of being submerged in a $\mathrm{K}_{2} \mathrm{PtCl}_{4}$ solution while rotating at $2400 \mathrm{rpm}$. The table below lists the hydrogen to palladium ratios obtained by integrating the $\mathrm{H}_{\text {des }}$ peak for each CV. (Right) TEM images of as prepared Pd nanocubes (top), Pd nanocubes after soaking in a $\mathrm{K}_{2} \mathrm{PtCl}_{4}$ solution for 5 min (middle), and 24 hours (bottom).

During galvanic replacement reactions of Pd with $\mathrm{Pt}$, the nanocube morphology changes from a cube shaped to a nanocage with a PtPd alloy composition, or in the case of a continuous growth of a Pt shell, the size of the nanocube increases. ${ }^{4}{ }^{5}$ Here, nanocube morphology and size is retained even after continuous soaking of $\mathrm{Pd}\{100\} \mathrm{NCs}$ in $\mathrm{K}_{2} \mathrm{PtCl}_{4}$ as can be seen in the TEM images on the right of Figure S1. These data clearly show that our Pt treatment does not affect the broader Pd $\{100\}$ volume and is limited to the surface.

\section{Additional Elemental and Microscopic Analysis.}

(a)

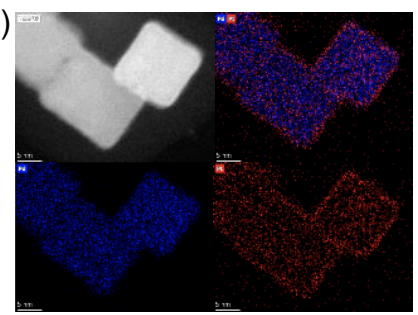

(e)

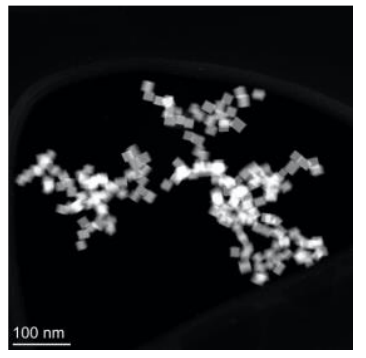

(b)

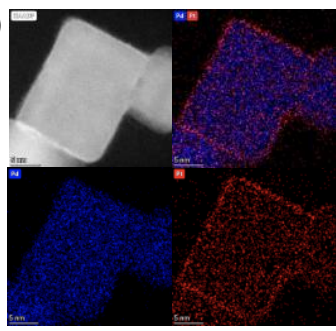

(f)

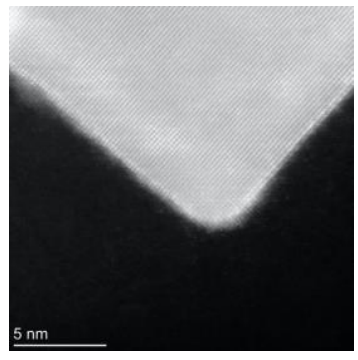

(c)

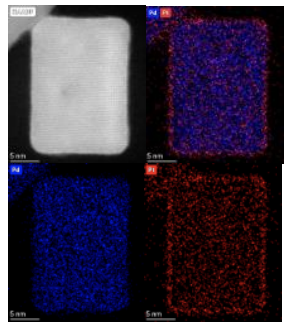

(g)

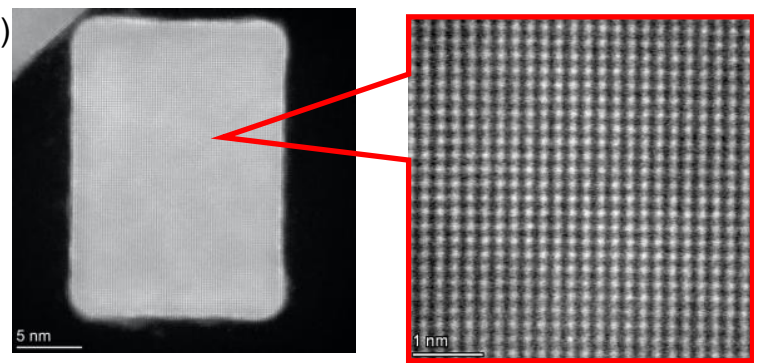

(d)

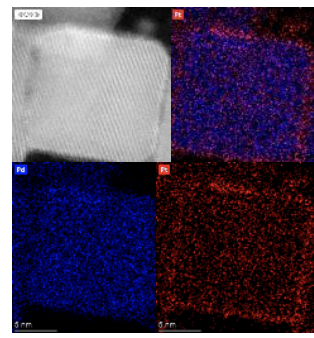

$5 \mathrm{~nm}$

(1)




\section{Hydrogen absorption/desorption isotherms and hysteresis.}
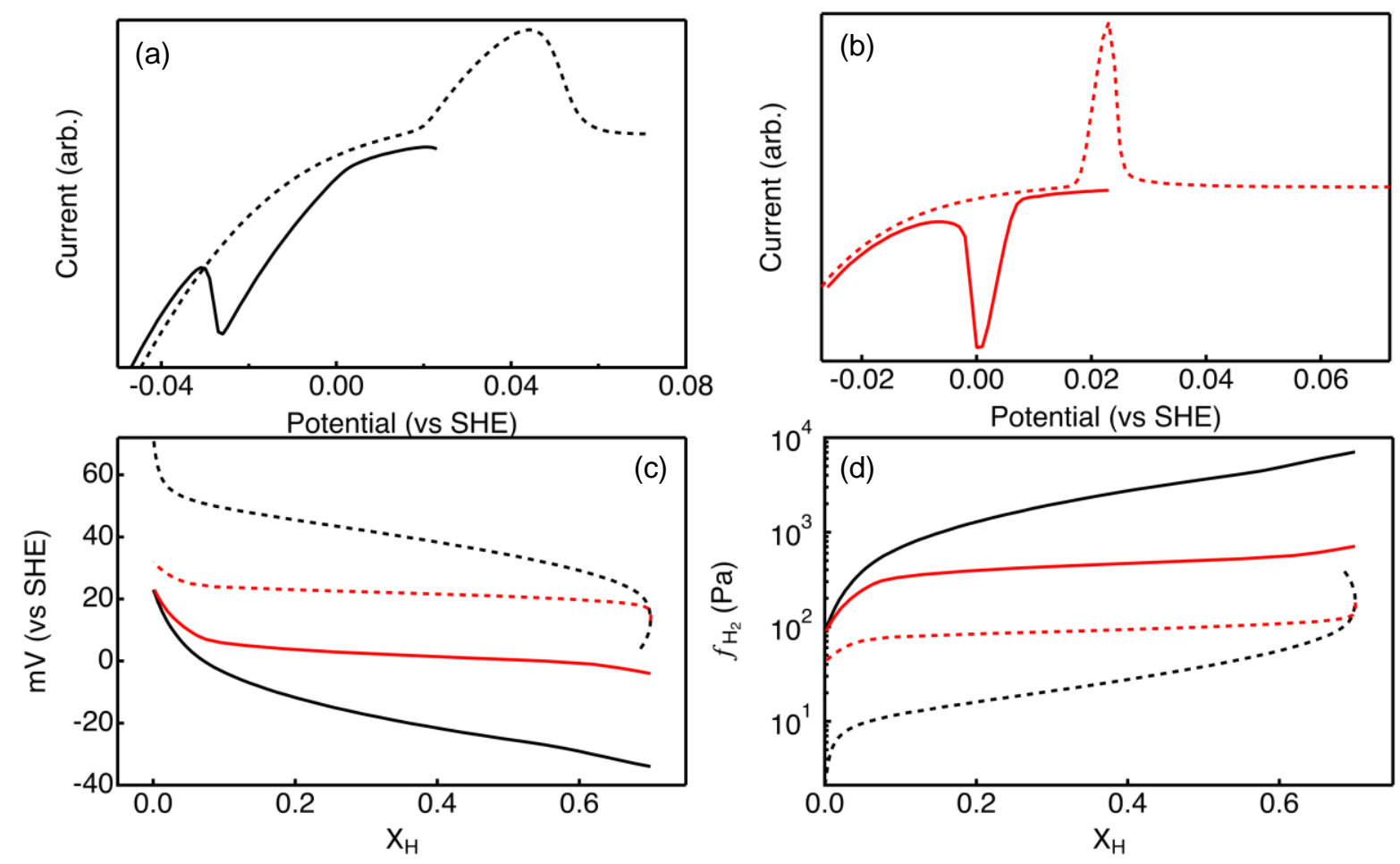

Figure S3. Electrochemical measurements of hydrogen absorption and desorption hysteresis and isotherms. (a) Linear sweep voltammogram of Pd nanocube electrode for hydrogen absorption (solid) and desorption (dashed) at $0.5 \mathrm{mV} / \mathrm{s}$ in $0.5 \mathrm{M} \mathrm{H}_{2} \mathrm{SO}_{4}$. (b) LSVs for the same electrode treated with a 5 min soak in $\mathrm{K}_{2} \mathrm{PtCl}_{4}$. (c) Absorption isotherm for the same data in Figure S3 (a) and (b). (d) Absorption isotherms for the same data in Figure S3 (c) plotted against the equivalent hydrogen fugacity.

The hysteresis between $\mathrm{H}_{\text {abs/des }}$ is both a benchmarking factor for hydrogen storage materials and a diagnostic of the $\mathrm{H}_{\text {abs/des }}$ mechanism. The hysteresis between hydrogen absorption and desorption arises from the energy barrier from coherency stresses during the phase transformation. ${ }^{6}$ Lattice dislocations can alleviate the energy barrier by facilitating incoherent phase transformations which reduce the $\mathrm{H}_{\mathrm{abs}} / \mathrm{des}$ hysteresis. To assess the hysteresis for our Pd and Pd@Pt nanocubes, we performed an analysis previously reported by Zalineeva et al. ${ }^{2}$ Electrochemical $\mathrm{H}_{\mathrm{abs}} / \mathrm{des}$ data were collected through linear sweep voltammograms at slow scan rates $(0.5 \mathrm{mV} / \mathrm{s})$ for Pd and Pd@Pt

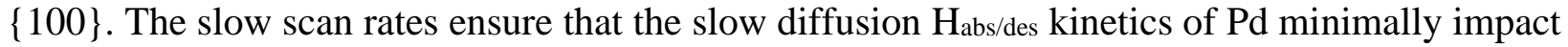
these results. From these LSV curves, the data display the same characteristics discussed in the main text- accelerated hydrogen absorption for Pd@Pt compared to Pd where a clear separation between hydrogen absorption and desorption peaks is evident. To obtain absorption isotherms, these data were integrated and normalized to the maximum hydrogen absorption capacity- $0.7-$ and plotted against the reference potential. Voltage isotherms are shown in Figure S3c. From S3c, we measure the voltage hysteresis of Pd $\{100\}$ and Pd@Pt $\{100\}$ to be $60 \mathrm{mV}$ and $22 \mathrm{mV}$, respectively. Finally, we calculate the $\mathrm{H}_{\mathrm{abs} / \mathrm{des}}$ isotherms against the equivalent hydrogen fugacity through the relationship: $\boldsymbol{f}_{\boldsymbol{H}_{2}}=\boldsymbol{a}_{\boldsymbol{H}^{+}} \exp \left[\frac{-2 F E_{S H E}}{\boldsymbol{R} T}\right]$, where $\boldsymbol{a}_{\boldsymbol{H}^{+}}$is the proton activity, $\boldsymbol{F}$ is the Faraday constant, $\boldsymbol{E}_{\boldsymbol{S H E}}$ is the electrode potential against the standard hydrogen electrode, $\boldsymbol{R}$ is the gas constant, and $\boldsymbol{T}$ is temperature. These data are plotted in Figure S3d and agree well with previous reports of $\mathrm{H}_{\mathrm{abs} / \mathrm{des}}$ on similar palladium nanocubes. ${ }^{2}$ 
From Figure S3, the hysteresis of Pd@Pt $\{100\}$ is clearly reduced compared to Pd $\{100\}$, but the equilibrium potentials are nearly identical indicating no thermodynamic change in $\mathrm{H}_{\mathrm{abs} / \mathrm{des}}$. Analyzing the magnitude of the hysteresis $\left(\ln \left[\mathrm{H}_{\text {fabs }} / \mathrm{H}_{\text {fdes }}\right]\right)$, we find that $\operatorname{Pd} @ \operatorname{Pt}\{100\}=1.5$, which, while is considerably smaller than $\mathrm{Pd}\{100\}\left(\ln \left[\mathrm{H}_{\text {fabs }} / \mathrm{H}_{\text {fdes }}\right]=4\right)$, a value of 1.5 is in excellent agreement with previous literature for coherent phase transformations. ${ }^{6}$ Therefore, $\mathrm{Pt}$ does not appear to change the nature of the phase transition mechanism compared to bare $\mathrm{Pd}\{100\}$ (i.e. coherency is retained).

\section{Electrochemical $H_{a b s / d e s}$ cycling stability of Pd $\{100\}$ and Pd@Pt $\{100\}$.}

To probe the stability of the Pd $\{100\}$ and Pd@Pt $\{100\}$ nanocubes during $\mathrm{H}_{\text {abs/des, }}$ we performed a cycle stability test. Here, Pd $\{100\}$ and Pd@Pt $\{100\}$ electrodes were electrochemically charged with hydrogen to its maximum and discharged a total of 100 times. The cyclic voltammogram of $\mathrm{H}_{\mathrm{abs} / \mathrm{des}}$ before and after $100 \mathrm{H}_{\mathrm{abs} / \mathrm{des}}$ cycles is shown in Figure S4a for both electrodes. The CV's for the 100 cycle test itself are shown in Figure S4b. Note the different scan rates for the data collected in S4a compared to S4b.
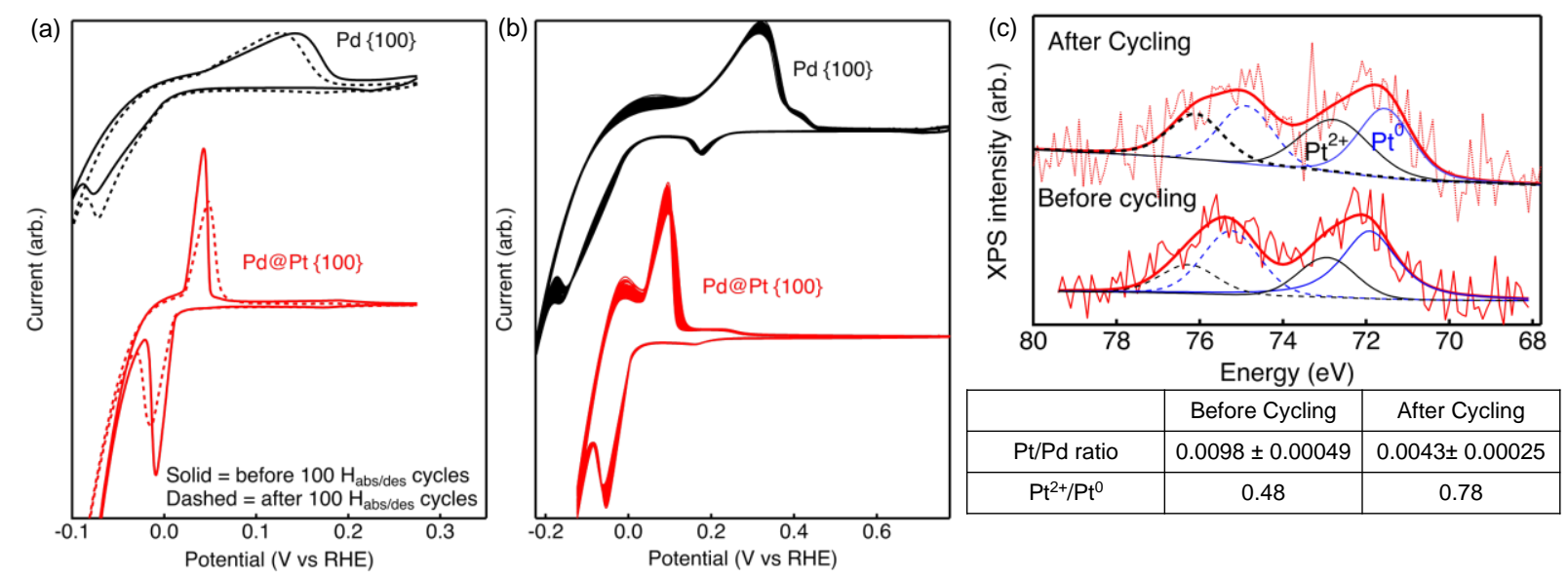

Figure S4. Electrochemical Habs/des Cycle Stability of Pd $\{100\}$ Pd@Pt $\{100\}$ electrodes. (a) Cyclic voltammograms of Pd $\{100\}$ and Pd@Pt $\{100\}$ before (solid) and after (dashed) 100 consecutive $\mathrm{H}_{\mathrm{abs}} / \mathrm{des}$ cycles. These data were collected at a scan rate of 10 mV/s. (b) 100 consecutive $\mathrm{H}_{\text {abs/des }}$ cycles for Pd $\{100\}$ and Pd@ Pt $\{100\}$. These data were collected at a scan rate of $100 \mathrm{mV} / \mathrm{s}$. (c) XPS measurements of the Pt $4 \mathrm{f}$ region before and after electrochemical hydrogen absorption cycles. The blue and black traces are the fits to the $\mathrm{Pt}^{0}$ and $\mathrm{Pt}^{2+}$ peaks, respectively, and the thick red trace is the envelope fit. The table below lists the $\mathrm{Pt} / \mathrm{Pd}$ and $\mathrm{Pt}^{2+} / \mathrm{Pd}^{0}$ ratios before and after electrochemical cycling.

From Figure S4a, both the Pd $\{100\}$ and Pd@Pt $\{100\}$ electrodes display acceptable hydrogen absorption/desorption reversibility. After $100 \mathrm{H}_{\text {abs/des }}$ cycles, each electrode displays a modest loss of capacity and a small shift in peak position. Notably, the Pd@Pt peak to peak separation for

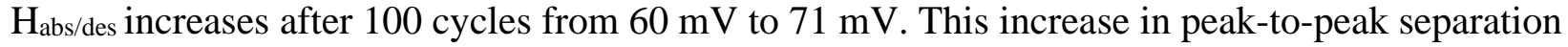
may be the electrochemical signature of $\mathrm{Pt}$ at the $\mathrm{Pd}$ surface migrating into the $\mathrm{Pd}$ nanocube

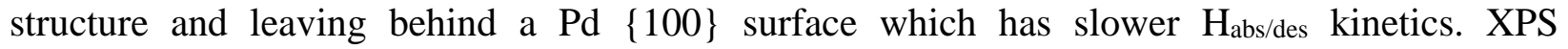
measurements before and after electrochemical cycling indicate a slight decrease in the $\mathrm{Pt} / \mathrm{Pd}$ composition, which, for a surface sensitive technique like XPS, is consistent with Pt migration into the Pd nanocube. We attempted to deconvolute the Pt XPS peaks into constituent $\mathrm{Pt}^{0}$ and $\mathrm{Pt}^{2+}$ which are shown in Figure S4c. From peak fitting deconvolution, the composition of the Pt $4 \mathrm{f}$ peak 
does not change significantly with cycling where the $\mathrm{Pt}^{2+} / \mathrm{Pt}^{0}$ ratio is 0.48 before cycling and 0.78 after suggesting that the Pt coordination environment is similar before and after cycling. Though, given the low signal-to-noise ratio of the Pt $4 \mathrm{f}$ spectrum in both measurements, these fits likely have a large associated error.

Finally, TEM images of Pd and Pd@Pt electrode-bound nanocubes were collected before and after 100 consecutive $\mathrm{H}_{\text {abs/des }}$ cycles (Figure S5). With the exception that the corners of the Pd nanocubes appear more rounded after cycling, the shape and size of the Pd and Pd@Pt $\{100\}$ particles are largely retained after $\mathrm{H}_{\mathrm{abs}} / \mathrm{des}$ cycling.
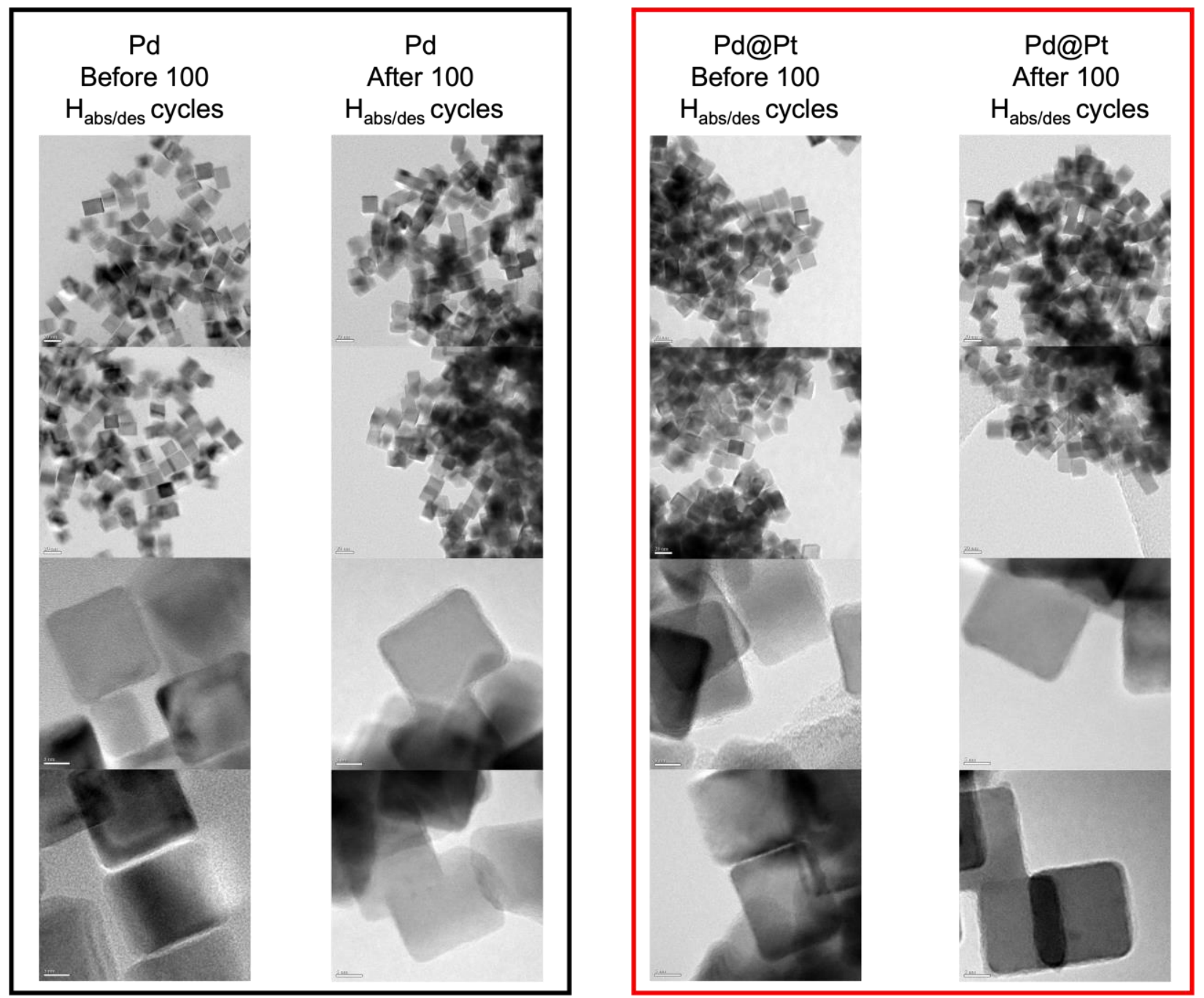

Figure S5. TEM images of cycled Pd nanocubes. TEM images of Pd $\{100\}$ and Pd@Pt $\{100\}$ before and after 100 consecutive $\mathrm{H}_{\mathrm{abs} / \mathrm{des}}$ cycles. These nanocubes were taken directly from the electrode surface. 


\section{Role of oxidant $\left(\mathrm{Na}_{2} \mathrm{PdCl}_{4}\right)$ on $\mathrm{H}_{\text {abs/des }}$ kinetics.}

$\mathrm{K}_{2} \mathrm{PtCl}_{4}$ is a strong oxidant and may affect the surface of the nanocubes simply by virtue of its redox potential $\left(\mathrm{Pt}^{2+}+2 \mathrm{e}^{-} \rightarrow \mathrm{Pt}^{0} E=1.2 \mathrm{~V}\right)$. To rule out the possibility that the enhanced kinetics we observe are simply an effect of exposure to a strong oxidant, we performed a control experiment where the nanocube electrode was exposed to $\mathrm{Na}_{2} \mathrm{PdCl}_{4}\left(\mathrm{Pd}^{2+}+2 \mathrm{e}^{-} \rightarrow \mathrm{Pd}^{0}, E=0.92 \mathrm{~V}\right)$ in the same way as the Pd@Pt electrode. The CVs of the Pd $\{100\}$ electrode before and after $\mathrm{Na}_{2} \mathrm{PdCl}_{4}$ exposure are shown in Figure S3. Here, it is clear from the near complete overlap between the two traces that the simple effect of exposure to an oxidant does not impact the $\mathrm{H}_{\text {abs/des }}$ kinetics.

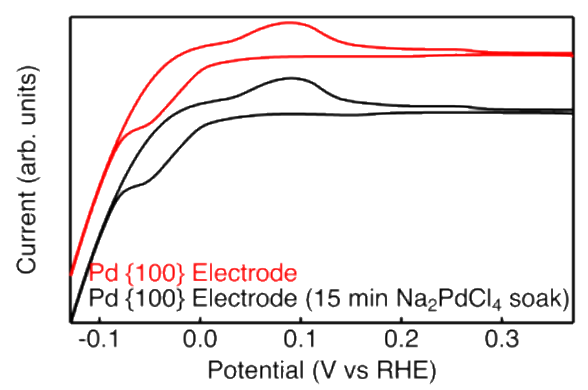

Figure S6. The effect of soaking the $\mathbf{P d}\{\mathbf{1 0 0}\}$ in $\mathrm{Na}_{2} \mathbf{P d C l}_{4}$. Cyclic voltammograms for a $\mathrm{Pd}\{100\}$ electrode in $0.1 \mathrm{M} \mathrm{H}_{2} \mathrm{SO}_{4}$ (black) before and after (red) exposure to $\mathrm{Na}_{2} \mathrm{PdCl}_{4}$.

\section{Unsupported Pd $\{100\}$ nanocube electrodes.}

To rule out the possibility that the other components of the composite electrode (conductive carbon and Nafion 117) are affecting the $\mathrm{H}_{\text {abs/des }}$ electrochemistry, we performed analogous electrochemical $\mathrm{H}_{\mathrm{abs}}$ /des experiments on unsupported Pd $\{100\}$ nanocube electrodes. In this data set, only $\mathrm{Pd}\{100\}$ nanocubes are deposited onto the surface of the electrode. Following the initial $\mathrm{CV}$, the electrode was exposed to $1 \mu \mathrm{M} \mathrm{K}_{2} \mathrm{PtCl}_{4}$. The nearly identical change in electrochemical

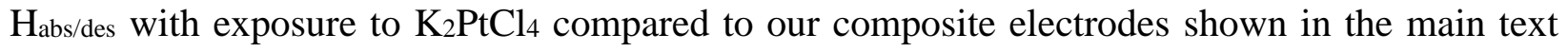
indicate that the other components of the composite electrode do not influence our findings.

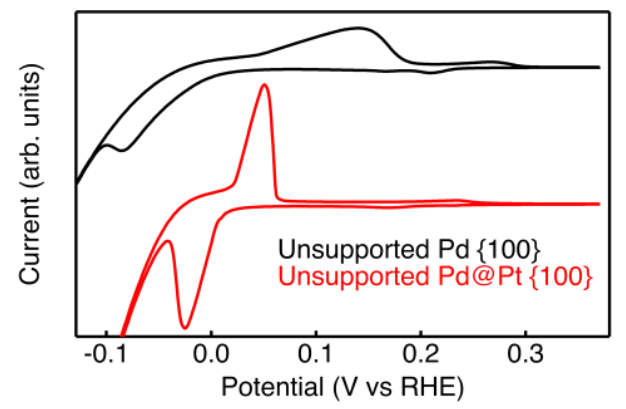

Figure S7. Unsupported Pd and Pd@Pt $\{\mathbf{1 0 0}\}$. Cyclic voltammograms for Pd $\{100\}$ and Pd@Pt $\{100\}$ physiosorbed to the surface of a glassy carbon electrode in $0.1 \mathrm{M} \mathrm{H}_{2} \mathrm{SO}_{4}$. 


\section{Mass Transport Considerations and Determination of Hydrogen Diffusion Coefficients.}

Under controlled hydrodynamic flow from a rotating disk electrode, the limiting-current from analyte flux (mass transport to the electrode surface) for a fast electron-transfer reaction is described by the Levich equation (equation 1).

$$
i_{L}= \pm 1.55 n F A D^{2 / 3} v^{-1 / 6} \omega^{1 / 2} c
$$

Here, $i_{L}$ is the mass transport limiting current, $n$ is the number of electrons transferred, $F$ is Faraday's constant, $A$ is the electrode surface area, $D$ is the analyte diffusion coefficient, $v$ is the kinematic viscosity, $\omega$ is the angular rotation rate, and $c$ is the concentration. For a $0.1 \mathrm{M} \mathrm{H}_{2} \mathrm{SO}_{4}$ solution at a rotation rate of $2400 \mathrm{rpm}$, the $\mathrm{H}^{+}$limiting current is >10 Amps. Compared to the maximum current that we measure for $\mathrm{H}_{\mathrm{abs}}(3 \mathrm{~mA})$, the solution-phase mass transport-limited current is well above the limiting process for $\mathrm{H}_{\text {abs. This }}$ comparison indicates that, while it is intuitive, the diffusion of $\mathrm{H} \bullet$ inside of $\mathrm{Pd}$ is not limited by the flux of $\mathrm{H}^{+}$to the $\mathrm{Pd}$ surface at any of our scan rates. Rather, the diffusion of hydrogen inside of palladium is analogous to an unstirred electrolyte solution. Under potentiodynamic conditions, this type of mass transport is described by Fick's second law. The time-dependent activity profile of a reversible redox couple in an undisturbed solution is:

$$
\frac{\partial c}{\partial t}=D \frac{\partial^{2} c}{\partial x^{2}}
$$

where $t$ is time since the beginning of a voltage pulse, $D$ is the analyte diffusion coefficient, and $x$ is the distance from the surface of the electrode. In a homogeneous system (i.e., a solvated molecule), the conditions necessary for Fick's law to be applicable require that the solution is quiet (not stirred). While the solution of our experiments is clearly stirred (laminar flow to the electrode surface from an RDE), the Pd nanocube is not 'stirred'. In our measurements, the Pd nanocube lattice starts as either vacant of hydrogen (for $\mathrm{H}_{\text {abs }}$ ) or alloyed with hydrogen (for $\mathrm{H}_{\mathrm{des}}$ ). The redox substrate in this analogy is the presence or absence of $\mathrm{H} \bullet$ in the Pd lattice. Since the Pd atomic arrangement is unchanged (except for a lattice expansion) with $\mathrm{Habs}_{\text {abes }}$, and the flux of $\mathrm{H}^{+}$is never limiting, the Pd 'solution' is quiet and undisturbed, and thus, $\mathrm{H} \bullet$ diffusion in Pd occurs under static conditions. The concentration of hydrogen is the molarity of $\mathrm{H} \bullet$ in the $\beta-\mathrm{PdH}$ phase (for $\mathrm{H}_{\text {des}}$ ) or the molarity of the holes of $\mathrm{H} \bullet$ in Pd that would otherwise be occupied by hydrogen. We calculate that, based on the stoichiometric ratio of $0.6: 1 \mathrm{H}: \mathrm{Pd}$ and a $3 \%$ Pd lattice expansion, the hydrogen concentration in $\beta-\mathrm{PdH}$ is $\sim 66 \mathrm{M}$. Since we observed no noticeable change in the hydrogen storage capacity with the addition of Pt to the surface, the same concentration was used for Pd@Pt as well.

Potentiodynamic experiments to determine the diffusion properties of a species near an electrified interface are common. The peak current in a CV or LSV is related to the diffusion coefficient of a redox-active substrate by equation 3 :

$$
i_{p}=0.4463 n F A c_{0} \sqrt{\frac{n F v D}{R T}}
$$


where $R$ is the gas constant and $T$ is the temperature in Kelvin. At room temperature, this is the well-known Randles-Ševčík equation. The dependence of $i_{p}$ on the square root of the scan rate is normal Cottrellian behavior and differentiates semi-infinite linear diffusion mechanisms from restricted diffusion mechanisms. In our measurements, we observe that the diffusion of $\mathrm{H} \bullet$ in Pd@Pt $\{100\}$ obeys standard Cottrellian behavior with a linear dependence of $i_{p}$ on the square root of the scan rate. The peak current of $\mathrm{H} \bullet$ diffusion in $\mathrm{Pd}\{100\}$, however, is consistently quadratic with scan rate. To account for this, and extract meaningful diffusion coefficients, we modified the equation with an additional power factor $(n)$ such that fitting the scan-rate-dependent $\mathrm{CV}$ data was performed with the equation 4 :

$$
i_{p, f i t}=0.4463 n F A c_{0} \sqrt{\frac{n F v D}{R T}}^{n}
$$

In the main text, this equation is represented as

$$
i_{p, f i t}=m(\sqrt{[V / s]})^{n}
$$

The temperature-dependent values of $n$ for $\mathrm{H}_{\text {des }}$ are shown in Figure S8.

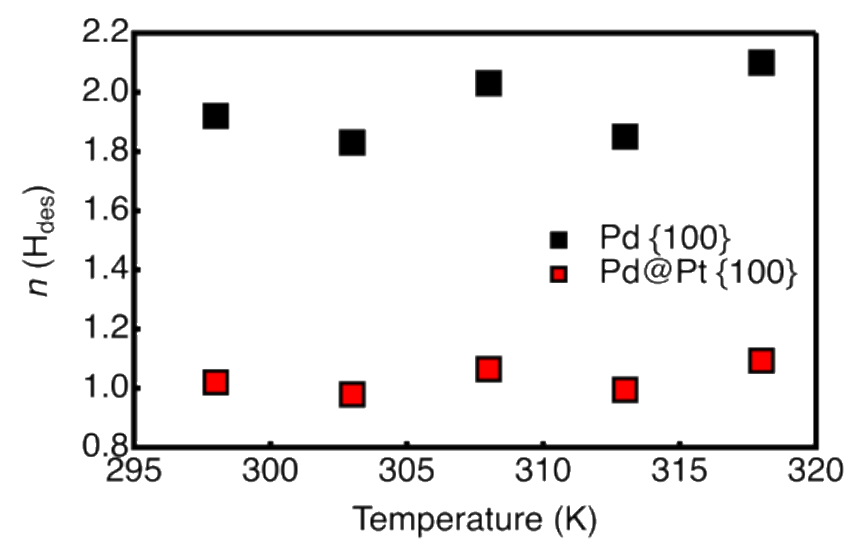

Figure S8. Plot of the observed power for the scan-rate-dependent peak current measurements for $\beta-\mathrm{PdH}$ (black) and $\beta-\mathrm{PdH} @ \mathrm{Pt}$ (red with black stroke) for $\mathrm{H}_{\mathrm{des}}$ at five different temperatures.

Here, an $n$ value of 1 indicates the expected linear dependence of $i_{p}$ on the square root of the scan rate. From the data in Figure S5, the floated power parameter, $n$, does not change meaningfully for either Pd $\{100\}$ or Pd@Pt $\{100\}$ at any temperature measured.

\section{Arrhenius Analysis.}

To quantify the energy barrier associated with $\mathrm{H}_{\mathrm{abs}} / \mathrm{des}$ in these $\mathrm{Pd}\{100\}$ and $\mathrm{Pd} @ \mathrm{Pt}\{100\}$ nanocube electrodes, we perform an Arrhenius analysis on the effective diffusion coefficient $\left(D_{e f f}\right)$ using equation 6 :

$$
D_{e f f}=D_{0} \times \exp \left[-\frac{E_{A}}{R T}\right]
$$


$D_{0}$ is the attempt frequency, $E_{A}$ is the activation energy, $R$ is the gas constant, and $T$ is the temperature. $E_{A}$ can be determined from the slope of a plot of $\ln \left(D_{\text {eff }}\right)$ vs. $1 / T$ (Figure 3e). All values of $E_{A}$ are shown in Table 1.

\begin{tabular}{|l|l|}
\hline \multicolumn{1}{|c|}{ Sample } & $E_{\mathrm{A}}(\mathrm{kJ} / \mathrm{mol})$ \\
\hline $\mathrm{H}_{\text {abs: }} \mathrm{Pd}\{100\}$ & $89 \pm 14$ \\
\hline $\mathrm{H}_{\text {des: }} \beta-\mathrm{PdH}\{100\}$ & $87 \pm 12$ \\
\hline $\mathrm{H}_{\text {abs: }} \mathrm{Pd} @ \mathrm{Pt}\{100\}$ & $1.8 \pm 0.53$ \\
\hline $\mathrm{H}_{\text {des }}: \beta-\mathrm{PdH} @ \mathrm{Pt}\{100\}$ & $1.1 \pm 0.13$ \\
\hline
\end{tabular}

Table S1. The values of $E_{\mathrm{A}}$ obtained from the fitting the temperature-dependent hydrogen diffusion coefficient data.

\section{References.}

1. Jin, M.; Liu, H.; Zhang, H.; Xie, Z.; Liu, J.; Xia, Y., Synthesis of Pd nanocrystals enclosed by $\{100\}$ facets and with sizes $<10 \mathrm{~nm}$ for application in CO oxidation. Nano Research 2010, 4 (1), 83-91.

2. Zalineeva, A.; Baranton, S.; Coutanceau, C.; Jerkiewicz, G., Octahedral palladium nanoparticles as excellent hosts for electrochemically adsorbed and absorbed hydrogen. Sci Adv 2017, 3 (2), e1600542.

3. Zalineeva, A.; Baranton, S.; Coutanceau, C.; Jerkiewicz, G., Electrochemical behavior of unsupported shaped palladium nanoparticles. Langmuir 2015, 31 (5), 1605-9.

4. Zhang, H.; Jin, M.; Liu, H.; Wang, J.; Kim, M. J.; Yang, D.; Xie, Z.; Liu, J.; Xia, Y., Facile synthesis of Pd-Pt alloy nanocages and their enhanced performance for preferential oxidation of CO in excess hydrogen. ACS Nano 2011, 5 (10), 8212-22.

5. $\quad$ Ataee-Esfahani, H.; Koczkur, K. M.; Weiner, R. G.; Skrabalak, S. E., Overgrowth Versus Galvanic Replacement: Mechanistic Roles of Pd Seeds during the Deposition of Pd-Pt. ACS Omega 2018, 3 (4), 3952-3956.

6. Griessen, R.; Strohfeldt, N.; Giessen, H., Thermodynamics of the hybrid interaction of hydrogen with palladium nanoparticles. Nat. Mater. 2016, 15 (3), 311-7. 